\title{
Response to Commentary: 'Myalgic Encephalomyelitis, Chronic Fatigue Syndrome, and Chronic Fatigue: Three Distinct Entities Requiring Complete Different Approaches'
}

\author{
Jessica E. Beilharz ${ }^{1} \cdot$ Scott J. Fatt ${ }^{1} \cdot$ Erin Cvejic $^{1,2} \cdot$ Andrew R. Lloyd $^{3} \cdot$ Ute Vollmer-Conna $^{1}$
}

Published online: 6 April 2019

(C) Springer Science+Business Media, LLC, part of Springer Nature 2019

We are surprised by the nature of the comments we have received primarily addressing the differences between myalgic encephalomyelitis (ME) and chronic fatigue syndrome (CFS), which was neither the focus, nor intended topic, of our article 'The invisible burden of chronic fatigue in the community: A narrative review' [1]. Rather, the intention of our review was to highlight the importance of investigating individuals in the community suffering from prolonged fatigue or chronic fatigue who have long been overlooked.

Does it really matter whether ME and CFS are synonymous labels or not? Both labels refer to an illness with heavily overlapping subjective symptom criteria and require a 6 months diagnosis. Given that both labels require the symptoms to be present for 6 months, individuals with the ME and CFS diagnostic label must initially have had a period of symptoms shorter than 6 months. Prolonged fatigue is defined as medically unexplained, debilitating fatigue that persists for 1 to 6 months [2]. Therefore, everyone who received a diagnosis of ME and CFS at 6 months must have suffered initially with prolonged fatigue, a condition that is poorly understood and understudied.

Syndromal diagnoses inevitably have somewhat uncertain diagnostic boundaries [3]. Therefore, there is good biological plausibility in the notion that some individuals will be subthreshold for the diagnostic label at one timepoint and then develop more severe or additional symptoms and, hence meet

Jessica E. Beilharz

j.beilharz@unsw.edu.au

School of Psychiatry, University of New South Wales, Sydney, Australia

2 School of Public Health, Faculty of Medicine and Health, University of Sydney, Sydney, Australia

3 Viral Immunology Systems Program, The Kirby Institute, University of New South Wales, Sydney, Australia the diagnostic criteria at another point in time. We explored this possibility in the now completed longitudinal dataset from the Dubbo Infection Outcomes Study (DIOS). In our review, the additional analysis of the DIOS dataset revealed a subgroup of individuals with an intermittent or relapsing pattern of fatigue (34/484 or 7\%). These individuals, while not scoring above the threshold score for clinically significant fatigue at every assessment point, evidenced a score at 6 months indicative of post-infective fatigue syndrome [1]. These preliminary results highlight the importance of also investigating individuals suffering from prolonged or chronic fatigue, and the fatigue trajectory that may lead to CFS and ME.

Finally, the merits or otherwise of cognitive behavioural therapy and graded exercise therapy for patients with CFS and ME are also not central of to our discussion. Rather, our review raises the question of whether timely intervention for those with prolonged or chronic fatigue might avert CFS and ME.

\section{Compliance with Ethical Standards}

Conflict of Interest The authors declare no conflicts of interest.

\section{References}

1. Fatt SJ, Cvejic E, Lloyd AR, Vollmer-Conna U, Beilharz JE. The invisible burden of chronic fatigue in the community: a narrative review. Curr Rheumatol Rep. 2019;21(2):5.

2. Fukuda $\mathrm{K}$, et al. The chronic fatigue syndrome: a comprehensive approach to its definition and study. Ann Intern Med. 1994;121(12):953-9.

3. Lloyd AR. Chronic fatigue and chronic fatigue syndrome: shifting boundaries and attributions. Am J Med. 1998;105(3a):7s-10s.

Publisher's Note Springer Nature remains neutral with regard to jurisdictional claims in published maps and institutional affiliations. 